Revista Iberoamericana, Vol. LXXIV, Núm. 225, Octubre-Diciembre 2008, 959-971

\title{
DESEOS DE ESTADOS QUEER \\ EN LA PRODUCCIÓN CRÍTICA LATINA DE LOS ESTADOS UNIDOS
}

\author{
POR \\ LÁZARO LIMA \\ Bryn Mawr College
}

I. Deseos DE Estado, Estados DE DESEO

Identificarse como "latino" en los Estados Unidos es instaurar una diferencia política radical que intenta contrarrestar las nocivas asociaciones socio-raciales que conllevan los apelativos “illegal”, “spic”, “greaser”, u otros aún menos apetecibles, tanto como las vivencias marcadas por la subalternidad política de los sujetos que habitan estas designaciones en la esfera pública de la contemplación nacional. Cuando se trata de latinos que a su vez se identifican como queer ${ }^{1}$ la aseveración es aún más complicada, ya que esta sobreidentidad calificativa se articula privilegiando el hecho de que lo significativo de semejante postura identificatoria es contrarrestar la heteronormatividad y sus diseños racializados en el entorno estadounidense. Entendido así, “ser” un latino queer implica una postura política que enfrenta una hegemonía que rehúsa calificar lo "latino”, mucho menos un "latino queer”, como algo cuyo significado podría designar algo más que su asociación con la “ilegalidad” en la esfera pública. Y si esta aseveración sorprende, vale recordar las observaciones del conocido periodista Frank Rich que estallaron en la blogosfera después de que aparecieran en las páginas del The New York Times: “Gay people [...] aren’t the surefire scapegoats they once were. Hence the rise of a jucier target: Hispanics. They are the new gays, the foremost political piñata” (12, énfasis mío).

Para Rich, la nueva “piñata” para golpear es la del cuerpo latino estadounidense cuya asociación con la "ilegalidad” lo consigna al espacio de lo abyecto en el imaginario social nacional, al espacio queer. Según este entendimiento de la latinidad en los Estados Unidos, a pesar de las diferencias afectivas, sexuales, tanto étnicas como lingüísticas, los latinos son como los gays; sujetos exluídos cuya "esencia”

1 La acepción más radical del término queer surge en los Estados Unidos con la publicación del libro del crítico Alexander Doty, Making Things Perfectly Queer. Doty prefiguró la utilidad política del término contra la heteronormatividad al señalar que "queer" designa "a flexible space for the expression of all aspects of non- (anti-, contra-) straight cultural production and reception” (3). 
transgrede los límites de la ley y el Estado. Entendido así, el concepto de la ciudadanía tanto como el de la ciudadanía cultural ${ }^{2}$ se descarta como un fundamento axiomático que instala la heterosexualidad como el espacio "natural" de la vivencia nacional y el origen del comportamiento cívico. En este ensayo me interesa explorar cómo el campo de los "estudios latinos" en los Estados Unidos se ha insertado dentro de la vanguardia de los "estudios queer" -creando aportes imprescindibles para un proyecto estrechamente asociado con la filosofía continental anglosajona- y, más allá de apropiarse de un discurso preexistente, ha reconfigurando los límites de dicho discurso bajo un proyecto que rehúsa entender el nacionalismo estadounidense como heteronormativo, ni como racial o lingüísticamente delimitado por las fronteras que configuran la nación. A continuación será útil señalar algunas de la pautas más significativas en torno a la crítica queer latina de los Estados Unidos y las líneas de investigación más recientes que empiezan a informar el futuro de la misma. ${ }^{3}$

\section{GENEALOGÍAS DEL SABER QUEER LATINO}

Las aproximaciones críticas y los debates de mayor repercusión que han informado el discurso queer latino de los Estados Unidos han sido el feminismo, los estudios lesbigay influidos por el constructivismo social que los siguió, y las aproximaciones posmarxistas elaboradas por los estudios culturales desde los ochenta hasta el presente. Sin embargo, el primer blanco de lo que se puede entender hoy como la crítica y la producción cultural queer latina en este país surge a partir del discurso crítico feminista chicano. La crítica y escritura feminista chicana, a veces imprecisamente asociada con lo que se ha llamado "the global Third Feminism", ${ }_{4}$ ofreció una metodología cuya base estableció el análisis de género junto con la cuestión de la raza y la subalternidad como los puntos de partida principales para la investigación de lo que hoy entendemos como "latinidad queer". ${ }^{5}$ Aunque los logros del feminismo occidental tanto como sus limitaciones han sido ya

2 Para Renato Rosaldo, “cultural citizenship”, lo que aquí traduzco como "ciudadanía cultural”, se refiere a la categoría de la ciudadanía "legal” tanto como a las vivencias y maneras de sobrevivir de sujetos subalternos que trabajan y viven en los Estados Unidos, sean legales o no.

3 Estas pautas estarán necesariamente delimitadas por cuestiones de espacio y por obsesiones críticas que no reflejan la diversidad del emergente campo de los estudios queer latinos.

4 Las distincciones entre el feminismo del "Primer mundo" y del "Tercero" se complicaron a partir de las intervenciones de Gloria Anzaldúa y Cherríe Morago, al resaltar éstas que el espacio "tercero" junto a la pobreza económica que lo sustenta también existía en los Estados Unidos. Para uno de los tratamientos más importantes del llamado "Third Feminsism”, véase Mohanty.

5 Me refiero aquí al importante trabajo de Juana María Rodríguez. Para Rodríguez "latinidad queer" se refiere a todas las modalidades posibles de la expresión sexual antinormativa. 
elaborados, ${ }^{6}$ es necesario reconocer que la labor crítica y la producción cultural de las chicanas Gloria Anzaldúa y Cherríe Moraga -entre varios otros escritores y críticos- ha reorientado el campo de los estudios latinos y los estudios queer, así como el feminismo en general. La publicación de This Bridge Called My Back. Writings by Radical Women of Color (1981) por Cherríe Moraga y Gloria Anzaldúa representa un hito fundamental en el desarollo del campo de estudios queer latinos. Al imbricar el análisis de la raza y la clase con el lesbianismo y el feminismo, se creó un modelo de práctica política alentada por la primera ola del feminismo estadounidense y las posibilidades abiertas por la militancia afroamericana. Con esta práctica política el feminismo chicano “queer” se inserta dentro de las varias tecnologías de identidad que habían orientado el discurso de la justicia y paridad social estadounidense, resaltando las correlaciones entre raza y clase social desde los setenta en adelante.

El conjunto de estas tecnologías de identidad abrieron el campo y sirvieron para revelar, por primera vez, cómo la cultura mayoritaria, tanto como la latina, habían privilegiado la heteronormatividad. En la temprana fecha de 1991 Carla Trujillo lo expresó así en su importante colección Chicana Lesbians: The Girls Our Mothers Warned Us About" "Our culture seeks to diminish us by placing us in the context of an Anglo construction, a supposed vendida to the race. More realistically, it is probably due to the fact that we do not align ourselves with the controlling forces of compulsory heterosexuality" (ix).

La elaboración del análisis de género y sexualidad en los estudios chicanos y latinos, pese a la vaticinadora observación de Trujillo, se desarrolló en torno a un esencialismo estratégico que resaltó la necesidad de proteger el espacio de producción e intervención política abierto por, como lo indica el subtítulo del texto de Moraga y Anzaldúa, las “radical women of color”. Con esta apertura política, el campo de lo que hoy podemos entender como "estudios latinos queer" entabla una significante querella en contra del sexismo y el racismo estadounidenses que a su vez anima la ampliación de lo que se había entendido como “Gay and Lesbian Studies” hasta ese momento. Esta apertura también crea una postura más crítica frente al estudio de las literaturas nacionales latinoamericanas, y los límites de los patrones establecidos por "las ficciones fundacionales", al cuestionar el papel de la heteronormatividad en la misma y los “sexilios” latinoamericanos en los Estados Unidos.

${ }^{6}$ Una de las críticas más desventadas al respecto fue elaborada por Elizabeth Fox-Genovese. Para la historiadora Fox-Genovese parece que el único “feminismo" de consecuencia fuera el de la vertiente anglo-sajona estadounidense y el feminismo continental.

7 Está importante colección de escritura creativa y estudios académicos ganó el prestigioso LAMBDA Book Award for Best Lesbian Anthology y el Out/Write Vanguard Award for Best Pioneering Contribution to the field of Gay/Lesbian Lifestyle Literature. 
No sorprende, entonces, que a partir de la década de los noventa empiezan a salir varios de los más importantes estudios sobre la cuestión queer en el contexto latinoamericano. ${ }^{8}$ Muchas de estas intervenciones, sin embargo, dejaron la cuestión queer latina fuera de los intereses inmediatos de los autores o editores, con las notables excepciones de las siguientes colecciones: ¿Entiendes? Queer Readings, Hispanic Writings (1995) de Emilie L. Bergmann y Paul Julian Smith, Hispanisms and Homosexualities de Sylvia Molloy y Robert McKee Irwin (1998), y Reading and Writing the Ambiente. Queer Sexualities in Latino, Latin American, and Spanish Culture de Susana Chávez-Silverman y Librada Hernández (2000). De la misma manera en que la publicación de This Bridge Called My Back reconfiguró el campo de estudios latinos y el emergente discurso latino queer, estas colecciones empezaron a establecer los parámetros para entender la cuestión queer latina en relación con los estudios latinoamericanos. La mayor parte de esta producción crítica se elaboró dentro del contexto de las humanidades y sus repercuciones fueron inmediatas aunque tardías en comparación con los estudios lesbigay que se habían elaborado a partir de la primera ola feminista en los Estados Unidos. Sin embargo, al contrario del constructivismo social que en gran parte organizó a los estudios lesbigay anglosajones en torno a cuestiones de acceso a circuitos de representación, visibilidad y el salir del armario (“coming out”), algunos de estos estudios insistieron en la necesidad de entender la sexualidad queer latina no sólo en su contexto estadounidense sino también en relación con sus diversos puntos de origen y sus particularidades locales.

En la colección ¿Entiendes?, por ejemplo, Arnaldo Cruz-Malavé examinó la falta de una explícita presencia lesbigay en el canon de la literatura puertorriqueña, dentro y fuera de la isla, para resaltar que aunque la literatura de la identidad lesbigay no sea explícita como tal, "the specter of homosexuality haunts Puerto Rico’s hegemonic discourse of national identity” (141). Al señalar cómo un escritor puertorriqueño canonizado como René Marqués rechazó las etiquetas de la identidad lesbigay de los Estados Unidos aunque el mismo Marqués fuera homosexual en un Puerto Rico ocupado, Cruz-Malavé empieza a leer la sexualidad latina dentro del entorno latinoamericano donde los "fundacionismos" nacionales decimonónicos crearon la base de los binarismos (hombre/mujer, casa/nación, civilización/barbarie, etc.) que establecieron la exclusión sociosimbólica que los sujetos subalternos y contranormativos han tenido que navegar. Escribiendo también en ¿Entiendes?, José Quiroga esboza lo que será uno de los aportes más importantes en su libro Tropics of Desire. Interventions from Queer Latino America (2000). Como Cruz-

8 Algunas de estas intervenciones críticas que sobresalen son de Bergmann y Smith; Foster y Reis; Foster; Balderston y Guy; Molloy y McKee Irwin; Chávez-Silverman y Hernández. Claro está, ésta no es una lista exhaustiva. 
Malavé, Quiroga desmiente la necesidad de adoptar los patrones de visibilidad y representación como el logro y la condición sine qua non del proyecto lesbigay constructivista. En Tropics of Desire, Quiroga entiende el deseo sexual como uno por un estado politíco y un estado del ser que a su vez transgrede las fronteras nacionales. (Por ejemplo, pensemos en las experiencias del "sexilio" transnacional de un Reinaldo Arenas). Es decir, Quiroga señala cómo no se puede despolitizar el campo de la producción cultural, no sólo en los Estados Unidos, sino en las Américas, al insistir en que el afán por la visibilidad y el acceso a circuitos de representación no marcan la meta o el límite del proyecto queer, ya que se trata de diferentes climas políticos en los cuales un estándar ecuménico no siempre ha sido posible o deseable. Esta crítica en contra de los imperativos de la "coming out narrative” estadounidense, que había dominado la política lesbigay, creó la posibilidad de ir más allá del discurso de identidad lesbigay anglosajón, en el cual "darle voz" al sujeto siempre ha implicado el riesgo de hablar por ella o por él. Aún más, el proyecto de Quiroga puso en evidencia los límites del discurso de identidad lesbigay que había considerado la identidad gay, lesbiana, o bisexual (sin vincular éstas con el transgenerismo) como una "subcultura"; algo que a su vez también ha implicado el riesgo de confundir la categoría de la cultura con el espacio político del Estado. Es decir, la elisión de "estados de deseo" por los "Estados nacionales" que legitiman a esos sujetos reproduciendo los valores del mismo. Entendidas así, las categorías de análisis de cultura y Estado ofrecen la falsa impresión de que las dimensiones culturales y materiales son intercambiables.

\section{LEGADOS}

Entrando ya en el nuevo siglo y frente al viejo imperativo lesbigay de la representación y la visibilidad, la crítica queer latina ha reconfigurado su relación con los discursos de identidad. Los varios proyectos identitarios influidos por el discurso de las teorías queer dan por sentado que la identidad es un efecto de la "actuación”, el llamado "performance of identity", 9 donde la actuacción no es expresiva de una integridad ontológica sino una postura performativa. No obstante, las consecuencias de estas actuaciones performativas de identidad queer latina, o su ausencia, siempre implican un costo.

En un bello artículo autoetnográfico deAlberto Sandoval-Sánchez, "Politicizing Abjection: In the Manner of a Prologue for the Articulation of AIDS Latino Queer Identities” (2005), el crítico precisa la utilidad de una aproximación a la

\footnotetext{
9 El estudio que inicia la línea performativa y antiesencialista en relación a los estudios latinos de los
} Estados Unidos es el de la crítica Karen Christian. 
performatividad "hecha carne y hueso"; es decir, desde la posición vivencial e ideológica. Refiriéndose a un impactante poema suyo que abre su meditación sobre el cuerpo queer latino, Sandoval-Sánchez afirma:

[The] reflection of a Latino gay man withAIDS constitutes a system of representation where the body is the foundation for a theoretical project in which writing, as a critical cultural practice, facilitates a politics of intervention and survival immersed in the deep and troubled muddy waters of abjection, an abjection entangled en $\mathrm{mi}$ mariconería y en mi Latinidad. And given that I do scholarship with my body, my writing is the umbilical cord to abjection, to my migrancy, to my mariconería, to my Latinidad, and to my survival. (548)

El "performance" de la latinidad queer toma la forma de un poema que es a su vez un testimonio. Significativamente, el poema está escrito en inglés y en español como en la cita que reproduzco. Es aquí, en el proceso de darle “carne y hueso” a la conceptualización de la identidad queer latina marcada por el sida, que el cuerpo recobra su historicidad al articular su valor y su densidad humana dentro del escenario nacional que lo considera como un sujeto abyecto y desechable. La intervención de Sandoval-Sánchez reconfigura el valor del cuerpo queer latino en el escenario nacional mediante la escritura y la memoria. Presagiando las observaciones de Frank Rich que abrieron mi reflexión, para Sandoval-Sánchez los latinos queer son “the foremost political piñata”. ¿Hasta qué punto, se pregunta SandovalSánchez, “do Latino/a queer cultural performances materialize a discursive site of/for abjection that menaces the homogeneity and stability of official hegemonic culture and identity and its anxieties that keep the queer, the AIDS survivor, the Latino/a migrant, the racial and ethnic Other locked in place?” (258). Esta pregunta se empezó a esbozar como pugna en contra de la heteronormatividad en la esfera pública en el importante libro de José Esteban Muñoz, Disidentifications. Queers of Color and the Performance of Politics (1999).

Disidentifications fue uno de los primeros textos en precisar la relación entre la etnicidad, la latinidad, los estudios queer, y la performatividad. Muñoz recalca la importancia de historizar el cuerpo queer latino fuera de los parámetros de las metodologías lesbigay. Muñoz describe su proyecto así, “[t]his study is informed by the belief that the use-value of any narrative of identity that reduces subjectivity to either a social constructivist model or what has been called an essentialist understanding of self is especially exhausted” (5). Poco después de la publicación de Disidentifications, los estudios de Muñoz continuaron reformulando la manera de entender la latinidad queer. Uno de ellos en particular merece mención aquí.

En "Felling Brown: Ethnicity...", Muñoz analiza la pieza teatral del chicano Ricardo Bracho, The Sweetest Hangover (and Other STDs), Muñoz empieza a 
proponer que la etnicidad queer latina se entienda en términos de "estructuras afectivas”. Para Muñoz,

[w] hiteness claims affective normativity and neutrality, but for that fantasy to remain in place one must only view it from the vantage point of US cultural and political hegemony. Once we look at whiteness from a racialized perspective, like that of Latinos, it begins to appear to be flat and impoverished. At this moment in history it seems especially important to position whiteness as lack. [...] Rather than trying to run from this stereotype, Latino as excess, it seems much more important to seize it and redirect it in the service of a liberationist politics. (70)

Aquí Muñoz desarrolla una tecnología de la identidad que reconoce cómo el poder de las anclas culturales crean comunidades latinas en un país que todavía no puede reconocer que la diferencia racial se sigue entiendiendo como el opuesto de un ideal nacional sin punto de origen fuera de la hegemonía que lo sustenta. Para Muñoz, entonces, el proyecto de la identidad queer latina sólo se puede llevar a cabo en la primera persona mediante la densidad de "afectos" individuales, expresados como comunales, que inventan mundos y reconfiguran las posibilidades de participar en la esfera pública sin capitular frente a los circuitos de representación que delimitan la forma y el contenido de la participación social. Muñoz resume su análisis de la obra de Bracho de la siguiente manera:

This analysis has posited ethnicity as a "structure of feeling," as a way of being in the world, a path that does not conform to the conventions of a majoritarian public sphere and the national affect it sponsors. It is my hope that thinking of latinidad in this way will help us better analyze the obstacles that must be negotiated within the social. (79)

La fuerza del proyecto de Muñoz reside en el reconocimiento de que la latinidad queer estadounidense, pese a las profundas diferencias afectivas y legales del sujeto latino frente a la nación, cambia los patrones de participación nacional en el enjambre de la esfera pública al rehusar el acceso que la normatividad nacional permite. De ahí la constante querella entre las prácticas de las identidades queer latinas fluidas y el contexto normativo nacional que exige que las identidades sean esenciales para manejarlas y delimitarlas a pesar de las necesidades o contextos locales de las diversas "latinidades" que integran las sujetividades queer latinas. Paradójicamente, algunos sectores de la actual vanguardia del discurso académico queer se han manifestado en contra de los mismos preceptos antinormativos que han exaltado. Quizás en su afán por liberarse de las connotaciones esencializantes del legado de los estudios lesbigay que fijaron los términos como "lesbiana”, "homosexual”, etc., este discurso ha delimitado el rol y la importancia de cualquier 
discusión cuya entrada a la política queer se base en la llamada "política identitaria racial”, o identity politics.

El crítico Hiram Pérez ha investigado las contradicciones inherentes en los modelos operativos dentro de la vanguardia de la queer theory que aparentan incluir la diversidad queer pero cuya práctica reniega la inclusion del queer latino o, como lo llama él, "the brown body", cuando este cuerpo político insiste en considerar la raza junto a la antinormatividad en los estudios queer. ${ }^{10}$ En su influyente artículo "You Can Have My Brown Body and Eat It, Too!” (2005), Pérez afirma que

[q]ueer theory is very particular about the kinds of trouble with which it troubles itself. The problem of race in particular presents queer theory with dilemmas over which it actively untroubles itself. [...] [T] he resistance within establishmentarian queer theory to thinking race critically, [is] a resistance that habitually classifies almost any form of race studies as a retreat into identity politics. This defensive posture helps entrench institutionally the transparent white subject [...]. Queer theorists who can invoke that transparent subject, and chose to do so, reap the dividends of whiteness. (171)

Pérez sostiene que el acusar a alguien de ser partidario de "identity politics", y las connotaciones con el esencialismo que este término ha arrastrado desde la primera ola del feminsimo, ha convertido la cuestión de la etnia y la raza en un tema ahistórico dentro de los estudios queer en los Estados Unidos. ${ }^{11}$ Si las afirmaciones de Pérez son representativas del estado actual de la vanguardia de la teoría queer, y creo que lo son, ¿̇a qué se debe esta reacción en contra de lo que es denigrado como identity politics por la vanguardia teórica queer y se vive como experiencia de exclusión por parte de las comunidades latinas subalternas? Antes de intentar responder esta pregunta, será útil perfilar algunas de las preocupaciones principales de esta vanguardia de estudios queer.

\section{FUTUROS QUEER}

Lee Edelman, en su impactante No Future: Queer Theory and the Death Drive (2004), inicia una nueva línea de investigación queer menos interesada en los efectos y afectos de las identidades queer y más en la figuración político-social de la

\footnotetext{
${ }^{10}$ Pérez nota con asombro un incidente que ocurrió en una conferencia en la Universidad de Michigan sobre el tema de las formas de "Queer Shame”. Relata cómo no sólo fue él el único invitado queer latino, sino que cuando observó públicamente las implicaciones de ello en una mesa lo acusaron de ser reaccionario y metodológicamente atrasado.

${ }^{11}$ Pérez afirma que en la conferencia su crítica cuestionando la falta de participación de "brown" queers fue considerada "paranoid criticism" y que sus formulaciones al respecto fueron caracterizadas como “too literal” y, por ende, démodées (179).
} 
niñez que los limita para una proyección y utilidad crítica. Para Edelman, hablando siempre dentro del contexto estadounidense, la “protección” de los “niños” debilita el proyecto queer democrático, tanto como cualquier polémica en busca de derechos para las minorías queer de toda índole, al postular que la encarnación literal de cualquier futuro nacional reside en la figuración heteronormativa de “la niñez”. Si la reproducción de valores nacionales se lleva a cabo a base de casamientos ventajosos a partir del siglo xIx en el contexto latinoamericano -como ya se ha señalado en diversos estudios “fundacionales” a los cuales ya he aludido- la niñez recobra la funcionalidad de estos casamientos al intentar asegurar que tanto los valores como el capital se acumulen para servir los intereses de las clases que, al sentimentalizar la niñez, convierten la misma en fetiche del futuro. Según Edelman,

[o]n every side, our enjoyment of liberty is eclipsed by the lengthening shadow of a Child whose freedom to develop undisturbed by encounters [...] with an "otherness" which its parents, its church, or the state do not approve, uncompromised by any possible access to what is painted as alien desire, terroristically holds us all in check and determines that political discourse conform to the logic of a narrative wherein history unfolds as the future envisioned for a Child who must never grow up. [...] The Child [...] marks the fetishistic fixation of heteronormativity: an erotically charged investment in the rigid sameness of identity that is central to the compulsory narrative of reproductive futurism. (21)

Para Edelman, si la imposibilidad de disfrutar de “nuestra” libertad tiene nombre, ese nombre, "the Child”, funciona metonímicamente como una inversión económica y afectiva en el futuro que prefigura la utilidad de la niñez como la condición que organiza ese futuro. A mi parecer, la fuerza de la propuesta de Edelman reside en el reconocimiento de que convertir a la niñez en la encarnación en el presente de un posible futuro implica nada menos que colonizar y oficiar ese futuro. Claro está, ni los intereses de Edelman ni su crítica explícita se desarrollan en torno a la comunidad queer latina de los Estados Unidos. Sin embargo, su polémica demuestra la necesidad de desmentir cualquier proyecto que basa su éxito en el simulacro de una ideación a la que nunca le ha interesado la diferencia sexual y mucho menos la diferencia queer latina.

Continuando la crítica de la heteronormatividad efectuada por Edelman, Judith Halberstam, en su In a Queer Place and Time: Transgender Bodies, Subcultural Lives (2005), añade e imbrica al transgenerismo como la postura más radicalizada en contra de una futuridad orientada por las doctrinas de la reproducción. ${ }^{12}$ Insertando

\footnotetext{
12 Vale mencionar que Halberstam también critica la "homonormatividad”, el afán lesbigay por la “respetabilidad” burguesa, a fin de señalar la complicidad de ciertos sectores de las comunidades gay-lésbicas.
} 
el transgenerismo en la discusión, Halberstam señala que "reproductive time and family time are, above all, heteronormative time/space constructs", añadiendo que esta condición de ser no es ni deseable ni posible para los que optan "to live outside reproductive and familial time as well as on the edges of labor and production" (10). Para Halberstam, ir en contra de esta futuridad reproductiva implica a la vez una crítica de clase. Ir en contra de esta hegemonía que concibe el futuro en términos heteronormativos es indiscutiblemente un proyecto utópico pero necesario. ¿Cómo imaginar un futuro queer en el cual el sujeto latino pueda insertarse sin abandonar las especificidades raciales y étnicas que demandan una paridad social y la liberación sexual? ¿Cómo imaginar, entonces, versiones del futuro no oficiadas por una hegemonía que rechaza los estados de deseos que rehúsan la reproducción heteronormativa? Semejante proyecto implica nada menos que la posibilidad de descolonizar ese futuro.

La crítica que Pérez ejerce sobre el estado actual de la vanguardia teórica queer no intenta explicar cómo se puede incluir la latinidad como elemento constitutivo de esta teorización o si semejante proyecto es deseable..$^{13}$ Para Pérez, parece que lo que él llama el "sujeto transparente", "the transparent subject" de los estudios queer, no puede admitir semejantes transformaciones sin arriesgar la desintegración de su rigor teórico. Sin embargo, estimo que quizás su intervención se puede entender como un intento de legitimar el valor de su importante empresa de la misma manera que los estudios lesbigay en su momento intentaron una política de asimilación cuya muestra de paridad social habían sido el casamiento legal, la adopción, la expansión de la definición de la famila, y otras posturas de asimilación burguesa. Con esto no quiero sugerir que se debe despolitizar el discuro teórico de la vanguardia queer estadounidense o dejar el futuro de la queer latinidad en manos incapaces. Al contrario, propongo una estrategia que pueda acomodar lo que Muñoz considera como la problemática central para el cuerpo político latino, “[a]t this moment in history it seems especially important to position whiteness as lack. [...] Rather than trying to run from this stereotype, Latino as excess, it seems much more important to seize it and redirect it in the service of a liberationist politics" (2000: 70). Y, ¿cómo se ha de manifestar esta "política de la liberación”? Para la crítica Juana María Rodríguez, una posible respuesta a esta pregunta se daría mediante intervenciones teóricas que no prescindan del activismo:

Activism is an engagement with the hauntings of history, a dialogue between the memories of the past and the imaginings of the future manifested through the acts of our own yearnings. It is an encounter with the ghosts that reside within and inhabit the symbolic and geographic spaces that shape our worlds. (37)

\footnotetext{
${ }^{13}$ Pérez no se refiere directamente al proyecto de Edelman en su artículo; aunque sí menciona el libro
} de Halberstam, Female Masculinity (1998). 
Para Rodríguez, esta dialéctica entre el presente y el pasado crea futuros, o en sus palabras, un futuro "manifested through acts of our own yearning".

En su importante intervención, Rodríguez elabora estos futuros mediate las historias de los queer latinos que aparecen en su análisis junto a su propia narrativa y sus anécdotas. Las teorizaciones propuestas por Rodríguez promueven un futuro antinormativo y descentrado en el cual las tecnologías de la identidad se extienden mediante el cibersexo, la producción cultural, el "performance", y la posibilidad de habitar estados de deseo hechos posibles mediante el bisturí: el transgenerismo. Aquí, el cuerpo queer latino se transgenera y se sitúa en el mismo espacio queer anteriormente delimitado por el discurso lesbigay que ideaba la diferencia sexual en términos tan esencialistas como las categorías estables que intentaba desconstruir.

\section{V. ¿Posidentidades?}

Estos futuros descolonizados propuestos por Edelman, Halberstam y por Rodríguez en el contexto latino apuntan a mundos posibles donde los estados de deseos del sujeto encuentran una expresión sin represalias dentro del Estadonación. A veces hay que recordar que, en nuestro afán por la aproximación crítica más sofisticada, perdemos el jouissance y las revelaciones que apuntan la misma producción cultural que estudiamos.

Por ejemplo, al leer el texto de Edelman, No Future, se me ocurrió que las brillantes propuestas antinormativas elaboradas porél ya habían encontrado expresión en la deliciosa novela de Mayra Santos-Febres, Sirena Selena Vestida De Pena (2000). En Sirena Selena se proponen “estados de ser” que intentan reconfigurar la relación del ciudadano, o ciudadano en proceso, frente al nacionalismo heteronormativo mediante el transgenerismo. El texto de Santos-Febres sobrepasa los límites del travestismo performativo de “dragas” y “dragos”, de la actuación performativa, al invocar un estado de ser anhelado en un futuro venidero mediante una reconfiguración corpórea. Esta reconfiguración corpórea conlleva la posibilidad de transformar el cuerpo político nacional, al complicar las funciones de la reproducción de valores heteronormativos fundamentados por una "política del futuro" en donde la niñez se presenta como la condición esencial del futuro, y la famila heterosexual como su punto de origen.

En Sirena Selena la niñez funciona como emblema de un futuro antiheteronormativo. Sirena, nacida niño y desamparada por su familia tanto como por el Estado, se gana la vida en las calles de San Juan satisfaciendo los placeres de hombres casados. La draga Martha Divine la rescata después de escucharla cantar en una cuneta cerca del bar El Danubio Azul, donde pronto la instala para 
que cante. La voz de Sirena, como la de las sirenas que seducen a Odiseo y a los que atienden a su canto, es casi tan prodigiosa como el falo que tiene que atar entre sus piernas para poder vestirse en los trajes que la convierten en la ilusión de la joven mujer que desea ser.

Lo radical de esta novela para mí, lo que me sorprende cada vez que la estudio en mis clases y en los pocos artículos que la iluminan, aparte de que Sirena rompa la normatividad de la casa de Hugo Graubel y su esposa Solange al seducirlo a él, es el hecho de que -pese al discuro del proteccionismo de la niñez que Edelman elabora como emblema de un futuro colonizado por la heteronormatividad reproductiva- nadie quiere proteger a Sirena por sí misma. A Sirena la desean como objeto de placer, como en el caso de Hugo y muchos otros, o como fin de lucro para Martha Divine que podrá completar su “operación” con las ganancias de los shows que propone, pero no como niña o niño que merezca la protección de su famila biológica o la famila nacional que es el Estado. ¿ Será por casualidad entonces que después de penetrar a Hugo con su tremendo falo Sirena desaparece de la faz de la tierra con las prendas de Hugo y los vestidos prestados de Martha? ¿Qué nos puede ofrecer Edelman y la vanguardia de la teoría queer que no nos ofrece Santos-Febres en Sirena Silena? Sirena Silena muestra que el estado, pese a los ademanes al contrario, no es expresivo sino performativo y que, por ende, hay que apropiarse del escenario antes de que caiga el telón. Sí, nosotros los latinos queer somos "the foremost political piñata".

Bibliografía

Christian, Karen. Show and Tell. Identity as Performance in U.S. Latina/o Fiction. Albuquerque: U of New Mexico P, 1997.

Cruz-Malavé, Arnaldo. "Toward an Art of Transvestism. Colonialism and Homosexuality in Puerto Rican Literature”. ¿Entiendes? Queer Readings, Hispanic Writings. Emilie L. Bergmann y Paul Julian Smith, eds. Durham: Duke UP, 1995. 137-167.

Doty, Alexander. Making Things Perfectly Queer. Interpreting Mass Culture. Minneapolis: U of Minnesota P, 1993.

Edelman, Lee. No Future. Queer Theory and the Death Drive. Durham: Duke UP, 2004.

Fox-Genovese, Elizabeth. Feminism Without Illusions. A Critique of Individualism. Chapel Hill: U of North Carolina P, 1991.

Halberstam, Judith. In a Queer Time and Place. Transgender Bodies, Subcultural Lives. Sexual Cultures. Nueva York: New York UP, 2005.

Female Masculinity. Durham: Duke UP, 1998. 
Muñoz, José Esteban. "Feeling Brown, Feeling Down: Latina Affect, the Performativity of Race, and the Depressive Position”. Signs: Journal of Women in Culture and Society XXXI/3 (2006): 675-688.

"Feeling Brown: Ethnicity and Affect in Ricardo Bracho's The Sweetest Hangover and Other STDs”. Theatre Journal LII/1 (March 2000): 67-79.

Disidentifications: Queers of Color and the Performance of Politics. Minneapolis: U of Minnesota P, 1999.

Mohanty, Chandra Talpade, Ann Russo, y Lourdes Torres, eds. Third World Women and the Politics of Feminism. Bloomington: Indiana UP, 1991.

Moraga, Cherríe, y Gloría Anzaldúa. This Bridge Called My Back. Writings by Radical Women of Color. Watertown: Persephone Press, 1981.

Perez, Hiram. "You Can Have My Brown Body and Eat It, Too!" What's Queer about Queer Studies Now? David L. Eng, Judith Halberstam y José Esteban Muñoz, eds. Social Text XXIII/84-85 Nos. 3-4 (Fall-Winter 2005): 173-191.

Quiroga, José. Tropics of Desire: Interventions from Queer Latino America. New York: New York UP, 2000.

Rich, Frank. "How Hispanics Became the New Gays”. New York Times (June 11, 2006): Sec. 4, 12.

Rodríguez, Juana María. Queer Latinidad. Identity Practices, Discursive Spaces. Nueva York: New York UP, 2003.

Rosaldo, Renato. "Cultural Citizenship, Inequality, and Multiculturalism”. Latino Cultural Citizenship. Claiming Identity, Space, and Rights. William V. Flores y Rina Menmayor, eds. Boston: Beacon Press, 1997. 27-38.

Sandoval-Sánchez, Alberto. "Politicizing Abjection: In the Manner of a Prologue for the Articulation of AIDS Latino Queer Identities". American Literary History XVII/3 (2005): 542-549.

Santos-Febres, Mayra. Sirena Selena Vestida De Pena. Barcelona: Mondadori, 2000.

Trujillo, Carla, ed. Chicana Lesbians: The Girls Our Mothers Warned Us About. Berkeley: Third Woman Press, 1991. 
